\title{
SEARCHING FOR $0-1$ ASSIGNMENT
}

\author{
THINH D. NGUYEN
}

\begin{abstract}
We are interested in the complexity of a problem involving linear $0-1$ assignments. We describe the relevant definitions for our problem in details.
\end{abstract}

\section{Notations AND PROBLEM STATEMENT}

First, it is convenient to delineate our problem on linear $0-1$ assignments as follows.

Suppose we have a linear equation in $n^{2}$ variables with integer (negatives allowed) coefficients of at most $m$ bits each.

Partition $\Pi_{1}$ the variables into $n$ disjoint sets of $n$ variables each.

Make another partition $\Pi_{2}$ the following way. Pick 1 variable in every disjoint set of variables in $\Pi_{1}$ form a new subset of variable of size $n$. Pick 1 variable in every disjoint set of variables in $\Pi_{1}$ not picked before to form a new subset of variable of size $n$. So we have another disjoint partition into $n$ disjoint sets of $n$ variables each.

Our problems are the following two similar ones.

Definition 1. (1a problem):

Is it NP-complete to decide if there is a valid (valid here means vanishing) 0/1 assignment with exactly $n$ of assignments to be 1 with exactly 1 assignment per disjoint set?

Definition 2. (1b problem):

Is it NP-complete to decide if there is a valid (valid here means vanishing) 0/1 assignment that satisfies exactly $n$ of assignments to be 1 with exactly 1 assignment per disjoint set $\Pi_{1}$ and with exactly 1 assignment per disjoint set $\Pi_{2}$ ?

We reduce subset sum to both of these.

\section{Reducing Subset Sum to Problem 1a}

Given a set of $m$ positive integers $A=\left\{a_{1}, \ldots, a_{m}\right\}$, and a positive integer $B$, the Subset Sum asks if there is a subset of $A^{\prime} \subseteq A$ a such that $\sum_{a_{i} \in A^{\prime}} a_{i}$.

We simply pick $n=(m+1)$ and build our set in this way:

- add $a_{1}, a_{2}, \ldots, a_{m}$

- add $m$ zeros

- add the only negative number $-B$

- "fill" the other remaining $n^{2}-2 m-1$ elements with $C=\sum_{a_{i}}+B+1$

Key words and phrases. assignment, integer, programming, solution, subset, sum. Perebor. 
$(\Rightarrow)$ If the original subset sum has a solution $A^{\prime}$ then it is sufficient to use this partition $\Pi_{1}$

$$
\begin{gathered}
{\left[\begin{array}{ccccccc}
a_{1} & C & C & \ldots & C & 0 & C \\
0 & a 2 & C & \ldots & C & C & C \\
\ldots & & & & &
\end{array}\right]} \\
{\left[\begin{array}{cccccc}
C & C & \ldots & 0 & a_{m} & C \\
C & C & \ldots & C & C & -B
\end{array}\right]}
\end{gathered}
$$

and for each "row" pick $a_{i}$ if $a_{i} \in A^{\prime}, 0$ otherwise (and pick the final $-B$ )

$(\Leftarrow)$ If there is a partition $\Pi_{1}$ with a vanishing $0 / 1$ assignment then note that:

(i) none of the $C$ s can be picked (they are too large)

(ii) at least one row must contain all elements different from 0 (because there are $m+1$ rows and only $m$ zeros); so at least one nonzero element must be picked

(iii) in every case the $-B$ element must be picked to vanish the sum;bri

So the elements $a_{i}$ picked, no matter how the elements are partitioned between the rows, sum exactly to $B$; i.e. they are a valid solution for the original Subset Sum problem.

\section{Reducing to Problem 1b}

The $(\Leftarrow)$ direction it uses only the constraints on $\Pi_{1}$ which are the same of case 1a (exactly 1 "pick" from each row).

The $(\Rightarrow)$ direction is also still valid; intuitively, just build the main diagonal of $\Pi_{1}$ with the elements of $a_{i} \in A^{\prime}$, the element $-B$, while the remaining elements of the diagonal are 0 s.

For example if $m=5$ and the solution of the subset sum is $A^{\prime}=\left\{a_{1}, a_{2}, a_{4}\right\}, \Pi_{1}$ is:

$$
\left[\begin{array}{cccccc}
a_{1} & 0 & C & C & C & C \\
C & a_{2} & 0 & C & C & C \\
C & C & 0 & a_{3} & C & C \\
C & C & C & a_{4} & 0 & C \\
C & C & C & C & 0 & a_{5} \\
C & C & C & C & c & -B
\end{array}\right]
$$

In this way, we can build $\Pi_{2}$ setting the element $j$ of row $i$-th equal to element $j$ of column $i$ in $\Pi_{1}$. The assignment clearly vanishes both $\Pi_{1}$ and $\Pi_{2}$ and the constraints are met.

\section{Conclusion}

Garey and Johnson [1] shapes their theory based on previous primal works of Cook, Levin and Karp. Johnson [2] moves on with the guide to this theory. As long as we study a mathematical conjecture, we should encourage ourselves of having enough labouring hours on popular maths books like these. Then, reading some articles on theory of computing like [3] is a good practice. Only after that, could we think of the ultimate final for all mathematics sciences.

\section{REFERENCES}

1. Michael R. Garey, David S. Johnson, Computers and Intractability: A Guide to the Theory of NP-Completeness

2. David S. Johnson, The NP-Completeness Column: An Ongoing Guide. pp.393-405 
3. Phan Dinh Dieu, Le Cong Thanh, Le Tuan Hoa, Average Polyno-mial Time Complexity of Some NP-Complete Problems, Theor. Comput. Sci. 46(3): pp.219-237 (1986)

Current address: Department of Mathematics, Moscow State University

Email address: kosmofarmer@yandex.com 